\title{
Education initiatives in the Trento Proton Therapy Center experimental area
}

\author{
Benedetto Di Ruzza ${ }^{a, *}$ \\ ${ }^{a}$ Trento Institute for Fundamental Physics and Applications (TIFPA-INFN), \\ Via Sommarive 14, 38123 Trento, Italy \\ E-mail: benedetto.diruzzadtifpa.infn.it
}

The experimental area of the Trento Proton Therapy Center (Trento, Italy) is a test-beam area devoted to research activities on particle physics sensors and biophysics. In this area particle physics sensors for medical or space applications can be tested using a proton beam with energy between $70 \mathrm{MeV}$ and $230 \mathrm{MeV}$. This area is also specially suitable for education initiatives addressed to both students or general public because in this space all the elements of a complex test-beam facility are accessible to the visitors: beam transport magnets, beam monitor devices, dose measurement devices, DAQ systems, target experimental sensors and remote monitoring systems for sensors. In this publication will be presented a description of the Trento Proton Therapy Center accelerator, the experimental area and also the public success obtained there during the Trento Smart City Week 2019 initiative in September 2019.

40th International Conference on High Energy physics - ICHEP2020

July 28 - August 6, 2020

Prague, Czech Republic (virtual meeting)

*Speaker 


\section{Introduction}

The experimental area of the Trento Proton Therapy Center (TPTC)[1] is a test-beam area reserved for non-clinical activities inside the medical facility. Generally only researchers can enter this area for physics, biophysics and radiological experiments and measurements, but it is also perfect place for education initiatives.

\section{The Trento Proton Therapy Center}

The Trento Proton Therapy Center (TPTC) is one of the only three medical facilities for hadron therapy operating in Italy. It is located in Trento and it is operated by the Azienda Provinciale Servizi Sanitari (APSS)[2].

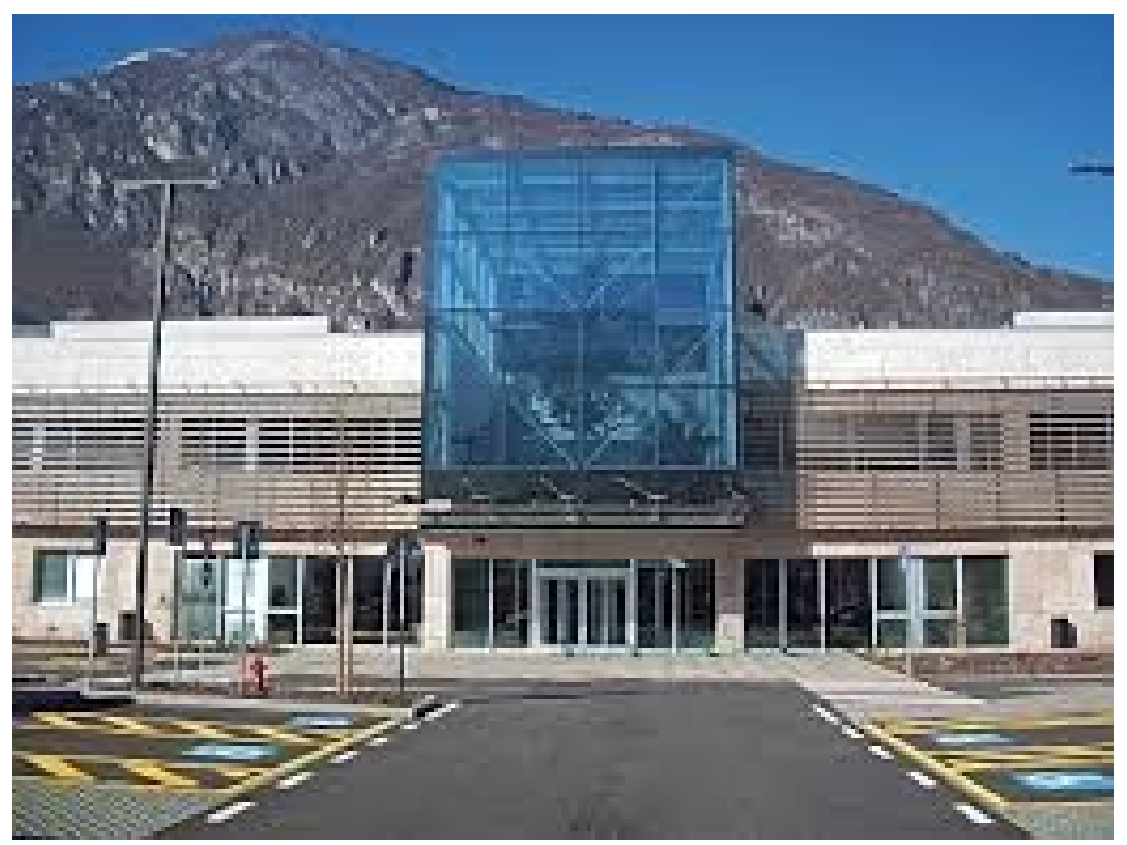

Fig. 2.1: The Trento Proton Therapy Center.

In the TPTC only protons are used for cancer therapy. Clinical activity started in 2014 and since then more than 1300 oncological patients were treated. The facility is equipped with two gantry rooms for patient treatment and a experimental area for physics and biophysics experiments. The experimental area is operated by the TIFPA-INFN [3] (the Trento center of the Italian National Institute for Nuclear Physics (INFN)[4]) in collaboration with the APSS and the IBA Company[5]. This area is used exclusively for non clinical activities such as physics, biophysics and radiological studies, tests and irradiations on cells or sensors. The parameters of the proton beam available in this area are well defined [6] and users can perform experiments in this area submitting requests to a PAC Committee [7]. 


\subsection{The proton source system}

The proton source system is composed of a cyclotron and a distribution line, that performs the proton beam distribution in one of the two gantry rooms during the clinical activities, or in the experimental area that is used only for no-clinical activities. Due to radiological restriction, the access in this area is allowed only to expert operators for short time when the beam is off. In September 2019, just before the Trento Smart City week 2019, the accelerator was exceptionally off for more consecutive days performing the annual review, and this allowed to open this area to small groups of guided visitors.

\subsubsection{The proton cyclotron}

The proton accelerator was built and is operated by the IBA corporation. It is a $105 \mathrm{MHz}$ cyclotron producing proton at the fixed energy of $228 \mathrm{MeV}$, then the beam energy can be lowered until $70 \mathrm{MeV}$ using a degrader. Maximum extraction current is $320 \mathrm{nA}[8]$.
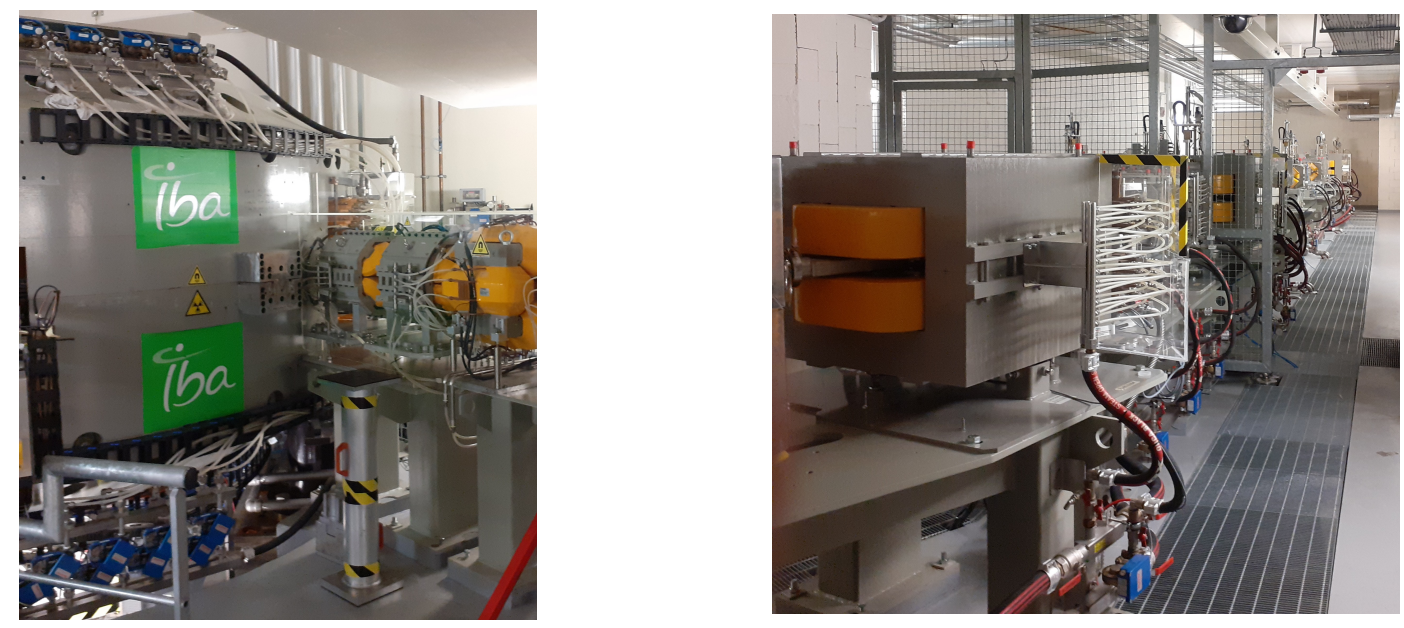

Fig. 2.2: The the cyclotron output point (left) and the beam distribution line (right).

\subsubsection{The distribution line}

After the accelerator the beam is bent and focused, and then using devoted switches it is delivered to the desired area.
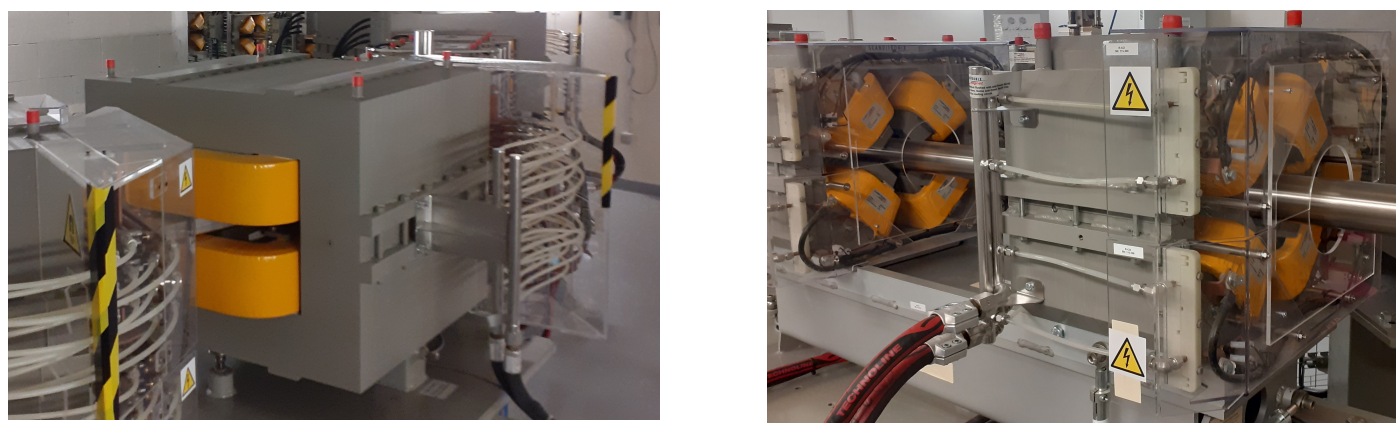

Fig. 2.3: Bending dipole (left) and focusing quadrupole (right) along the distribution line.

The radiation level in this area is generally quite high, and only the experts can enter here in special controlled situation. 


\subsection{The Gantry rooms}

The two gantry rooms are the place where every day patient treatment is performed. The two gantries can rotate 360 degree around the movable patient coach. Patient alignment is performed with two orthogonal movable Rx systems and then the patient position during the irradiation is monitored with a non-invasive system composed of three infrared cameras. In this area generally guided tours are organized weekly according to the clinical activity.
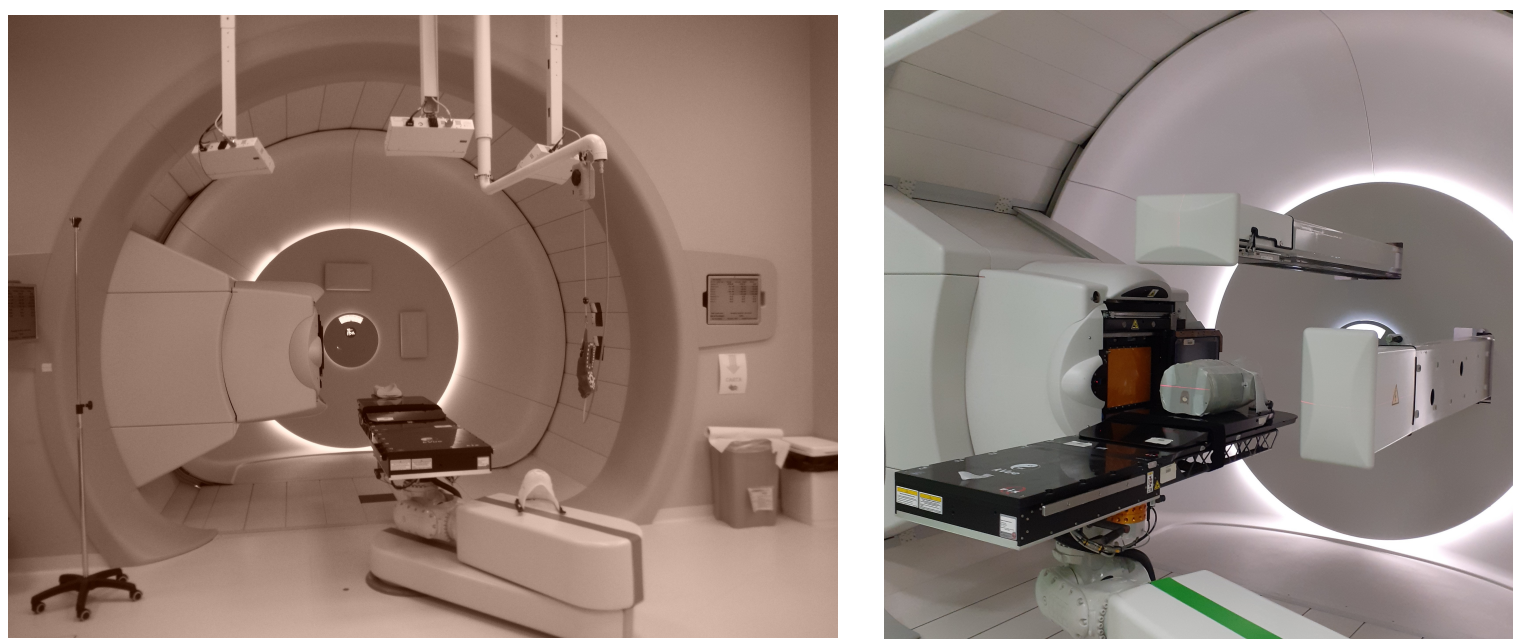

Fig 2.4: Gantry room overview (left) and patient alignment simulation (right).

\subsection{The Experimental area}

The experimental area is composed by two rooms: the cave, where two almost identical beamlines are present, and the multi-disciplinary laboratory, that is used also as control room for remote control of the instrumentation during the data taking. For practical reason one of the two beamlines, called zero degree, is used mainly for biophysics experiments while the thirty degree is used mainly for physics sensor experiment. Along the zero degree line is assembled the dual ring set-up [9] that is used for large area irradiations on cells culture and electronic devices.
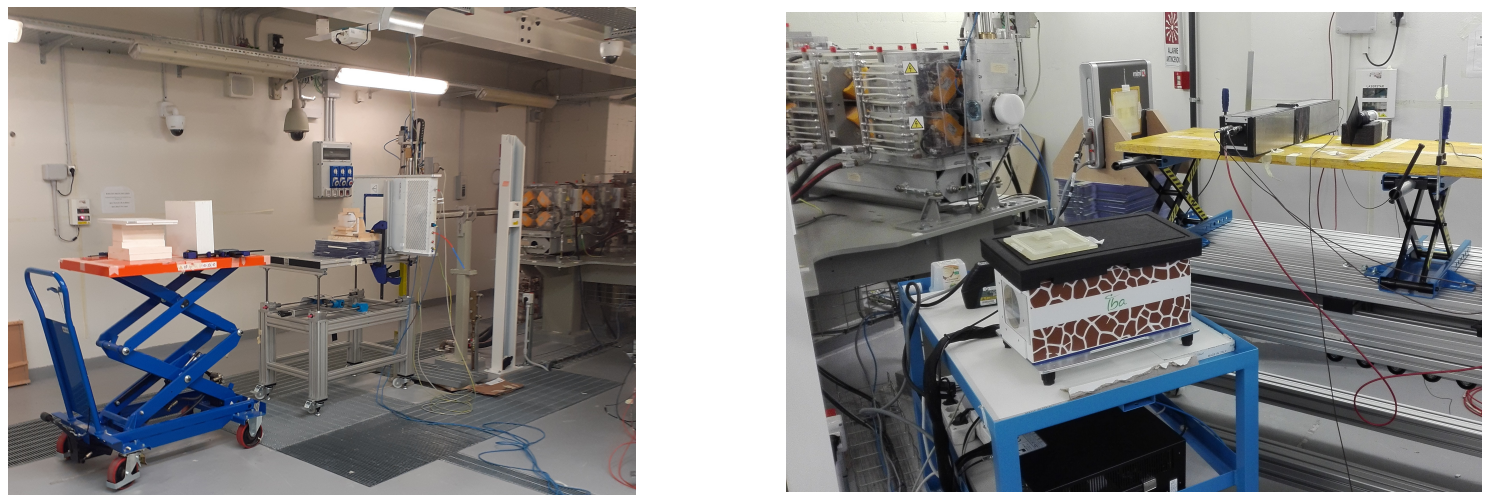

Fig 2.5: Biophysics (left) and physics (right) beamlines during the data taking. 
Since October 2020, thanks to the HERMES Bologna group, a new device is used for proton irradiation: the remote irradiation daisy, that allows to perform multiple irradiations remotely controlled without requiring the operators to enter the cave.

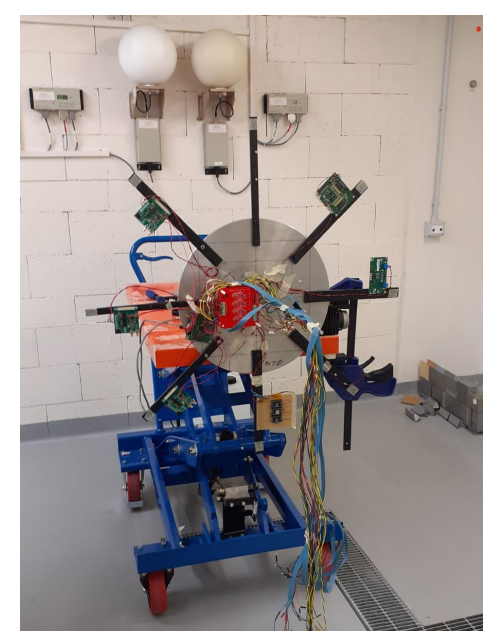

Fig. 2.6: The remote irradiation daisy with two neutron detectors on the back.

In the two rooms are present all the basic tools used in a test beam situation (beam monitor, trigger scintillators, moving stages etc.) that can be assembled on user request. The access in the experimental area is reserved to expert researcher and only in special situations visitor tours are organized.

\section{Trento Smart Week 2019}

Trento Smart Week 2019[9] is the 2019 edition of an event organized every year by the City of Trento, Trento Province with other local sponsor and partners. The motivation is that in recent years, the presence of digital services has become very pervasive in personal relationships, with the Public Administration and with companies. For this reason, it is important, if not urgent, to have awareness and the correct "instructions for use" for an effective and correct use of the new tools: rights and duties, reputation, importance of training and understanding.
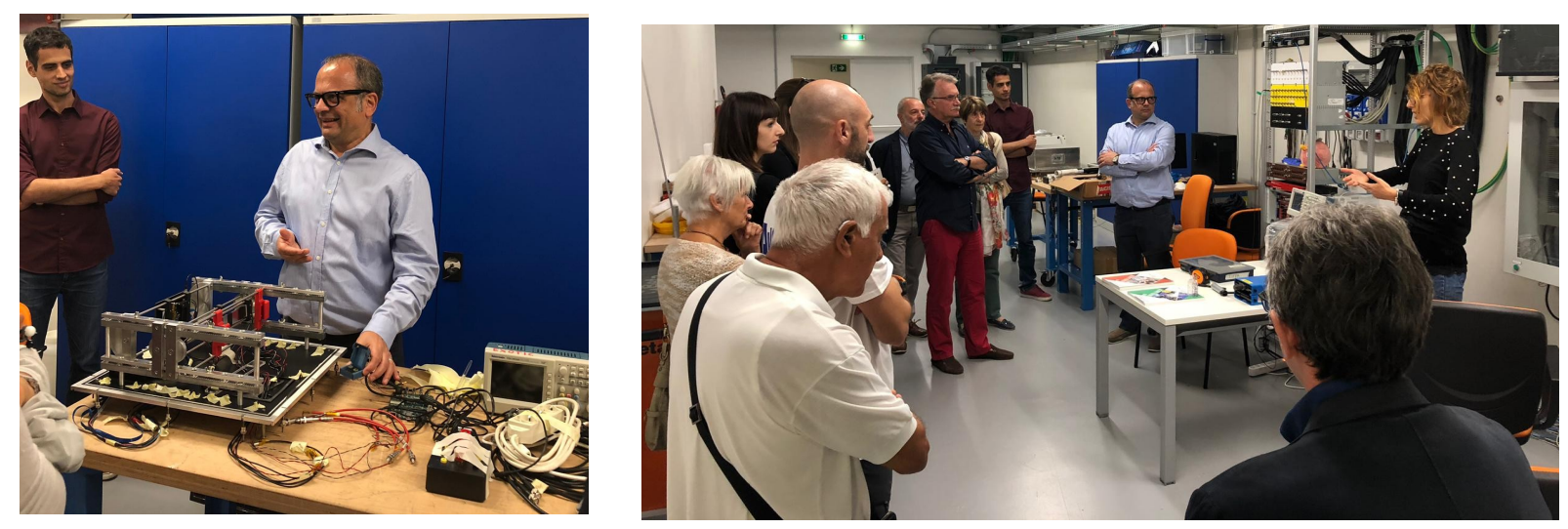

Fig. 3.1: Detectors exposition (left) and visitors attending the demonstration (right). 
In order to show how research can be complex, how works and what is the impact on everyday life for everybody, TIFPA-INFN, TPTC, the Physics Department of Trento University [11] and the Research Center Bruno Kessler Foundation [12] organized, inside this event, guided tours in the facility and public demonstrations of real research tools.

At the end of that day, around 60 visitors made the tour, all of them where enthusiastic of the tour, interested in the place and asked questions all the time.

\section{Conclusion}

Considering the feed-back of the visitors, the organizers had the evidence that a research facility is the best place to demonstrate how technology is affecting our every-day life and had the confirmation that is important for all the people get involved in the building of the future.

\section{References}

[1] Trento Proton Therapy Center - TPTC (Trento, Italy):

https://protonterapia.provincia.tn.it/eng\#

[2] Azienda Provinciale per i Servizi Sanitari - APSS (Trento, Italy): https://www . apss . tn. it

[3] TIFPA-INFN web site: https://www.tifpa.infn.it

[4] Italian National Institute for Nuclear Physics: https://home.infn.it/en

[5] Ion Beam Applications - IBA: https://iba-worldwide.com/proton-therapy

[6] F. Tommasino et al., Proton beam characterization in the experimental room of the Trento Proton Therapy facility, NIM A Volume 869, 11 October 2017, Pages 15-20 .

[7] User beam time applications: http://www.tifpa.infn.it/sc-init/med-tech/pbeam-research

[8] Yves Jongen, REVIEW ON CYCLOTRONS FOR CANCER THERAPY, Proceedings of CYCLOTRONS 2010, Lanzhou, China.

[9] F. Tommasino et al., A new facility for proton radiobiology at the Trento proton therapy centre: Design and implementation, Physica Medica 58 (2019) 99-106.

[10] Trento Smart City week 2019: https://2019. smartcityweek.it

[11] Physics Department University of Trento: https: //www.physics . unitn. it

[12] Research Center Bruno Kessler Foundation - FBK (Trento): https://www . fbk . eu/en 